Cardiol Young 2000; 10: 80

(C) Greenwich Medical Medıa Ltd. ISSN 1047-9511

\title{
Diary of Events
}

\section{0-11 February 2000}

Foundation Course in Cardiac Morphology, London.

Enquiries: Courses \& Conferences Office, Institute of Child Health, 30 Guilford St, London WCIN IEH, UK. Tel: +44 (0)207829 8692; Fax: +44 (0)207831 6902; e-mail: Courses@ich.ucl.ac.uk

\section{0-31 March 2000}

XXI Séminaire de Cardiologie Pédiatrique: Gènes, syndromes, séquences et tout ça. Paris, France.

Enquiries: Secrétariat du Service de Cardiologie Pédiatrique, Höspital Necker/Enfants-Malades, 149 rue de Sèvres, 75743 Paris cedex 15, France. Fax: (+33) 144494340

\section{5-7 April 2000}

$10^{\text {th }}$ Charleston Symposium on Congenital Heart Disease, Charleston, South Carolina,USA.

Enquiries: J Philip Saul, MD, Medical University of South Carolina, The Children's Heart Center, 165 Ashley Ave, PO Box 250915, Charleston, SC 29425, USA. Fax: (+843) 792 3284

\section{7-20 April 2000}

$9^{\text {th }}$ Annual Symposium "Cardiology in the Young", London. Enquiries: Courses \& Conferences Office, Institute of Child Health, 30 Guilford St, London WC1N 1EH, UK. Tel: +44 (0)20 7829 8692; Fax: +44 (0)20 7831 6902; e-mail: Courses@ich.ucl.ac.uk

18-20 May 2000

International Symposium on The Developing Heart, Prague. Enquiries: Czech Medical Association J.E. Purkyne, PO Box 88, Sokolská 31, 12026 Prague 2, Czech Republic. Tel: 420 2297 27 1; Fax: 4202294 610;

e-mail: senderova@cls.cz; www.biomed.cas.cz/fgu/cardiol/ dh2000.htm
14-17 June 2000

$35^{\text {th }}$ Annual Meeting Association for European Paediatric Cardiology, Strasbourg, France, 14-17 June 2000. Enquiries: Dr Bernard de Geeter, Cardiologie Pédiatrique et Frale, 3 rue Simonis, F-67100 Strasbourg. Fax: +33 (388) 3430 04; email: drdegeeter@mail.sdv.fr

29-30 June 2000

New Horizons in Fetal Cardiology, London.

Enquiries: Courses \& Conferences Office, Institute of Child Health, 30 Guilford St, London WC1N 1EH, UK. Tel: +44 (0)20 7829 8692; Fax: +44 (0)20 7831 6902; e-mail: Courses@ich.ucl.ac.uk

13-14 July 2000

Advanced Course in Cardiac Morphology, London.

Enquirtes: Courses \& Conferences Office, Institute of Child Health, 30 Guilford St, London WC1N 1EH, UK. Tel: +44 (0)20 7829 8692; Fax: +44 (0)207831 6902; e-mail: mailto:Courses@ich.ucl.ac.uk Courses@ich.ucl.ac.uk

12-15 November 2000

$73^{\text {rd }}$ Scientific Session of American Heart Association, New Orleans, LA, USA.

Enquiries: AHA, Scientific Meetings, 7272 Greenville Ave, Dallas, TX 75231-4596, USA. Fax: (+214) 373 3406; E-mail: scientificconferences@heart.org; www.americanheart.org/scien tific/confer/index.html

27-31 May 2001

$3^{\text {rd }}$ World Congress of Paediatric Cardiology \& Cardiovascular Surgery, Toronto, Canada, May 27-31 2001. Enquiries: Dr R M Freedom, Head, Div of Cardiology, Hospital for Sick Children, University of Toronto, Faculty of Medicine, 555 University Ave, Toronto, Ont. M5G 1X8, Canada. Fax: $+1-4168137547$ 